\title{
SKIN CHANGES IN NEONATES DURING EARLY NEONATAL PERIOD
}

\author{
Parag Sharma ${ }^{1}$, Abhiram Behera ${ }^{2}$
}

${ }_{1}^{1}$ Associate Professor, Department of Dermatology and Venereology, Terna Medical College, Nerul, Navi Mumbai. 2Statistician cum Lecturer, Department of Community Medicine, Terna Medical College, Nerul, Navi Mumbai.

\begin{abstract}
BACKGROUND

The early neonatal period is considered to be the first 7 days of life after birth. The skin undergoes a variety of changes during this period, which reflects the neonates' functional adaptability to its new environment. These changes resolve spontaneously within a few weeks and are regarded as physiological. Skin changes other than physiological are called pathological. These pathological changes are either transmitted from the parents or acquired from the external environment.

The objective of this study is to determine the relative incidence of physiological and pathological skin changes, their relation to maternal and neonatal factors and the time of their appearance and disappearance in this part of the world.
\end{abstract}

\section{MATERIALS AND METHODS}

1000 live-born babies delivered in labour room over a period of 1 year and who could remain in the hospital for at least 7 days were selected for the present study. Babies were followed up daily till the next 7 days.

\section{RESULTS}

1000 neonates developed 6905 skin changes during their early neonatal period at an average of 6.9 lesions per neonate. Only 2 of them required immediate intervention. There were 5820 (84.3\%) physiological skin changes and 1085 (15.7\%) pathological skin changes. Pathological skin changes include 366 (5.3\%) infectious, 514 (7.4\%) non-infectious and 205 (3\%) developmental defects. Amongst physiological skin changes, physiological desquamation of skin was the most common and physiological jaundice was the least common. Amongst infectious skin disorders, ophthalmia neonatorum (23.3\%) was the most common and breast abscess $(0.1 \%)$ was the least common. Amongst non-infectious skin disorders, miliaria crystallina (35.3\%) was the most common and sclerema neonatorum (0.1\%) was the least common. Amongst developmental defects, Salmon patch (19\%) was the most common and Spina bifida $(0.1 \%)$ was the least common. Out of 5820 physiological skin changes, maximum (3716) appeared on the first day and minimum (109) appeared on the seventh day of life. Out of 890 infectious and non-infectious skin changes, the lesions appeared with an equal frequency throughout the 7 days.

\section{CONCLUSION}

Physiological skin changes were found more commonly than pathological skin changes, and the ratio between physiological to pathological skin changes was 5.8: 1.1. Physiological skin changes varied significantly with neonatal and maternal factors, while most of the pathological skin changes did not vary with the above factors. The parents can be assured that their babies will develop some skin lesions during their early neonatal period. Most of them will disappear spontaneously or with minimal treatment. Rarely, immediate intervention is required.

\section{KEYWORDS}

Physiological and Pathological Skin Changes, Neonatal and Maternal Factors, Day of Appearance.

HOW TO CITE THIS ARTICLE: Sharma P, Behera A. Skin changes in neonates during early neonatal period. J. Evolution Med. Dent. Sci. 2017;6(93):6695-6702, DOI: $10.14260 /$ jemds/2017/1450

\section{BACKGROUND}

The early neonatal period is considered to be the first 7 days of life after birth. The skin undergoes a variety of changes during this period, which reflects the neonates' functional adaptability to its new environment. These changes resolve spontaneously within a few weeks and are regarded as physiological.(1) Skin changes other than physiological are called pathological. These pathological changes are either transmitted from the parents or acquired from the external environment.

'Financial or Other Competing Interest': None.

Submission 30-10-2017, Peer Review 23-11-2017,

Acceptance 29-11-2017, Published 11-12-2017.

Corresponding Author:

Dr. Parag Sharma,

Flat No. 305, Dharti Complex,

Plot 60/61, Sector-18,

Kamothe, Navi Mumbai-410209.

E-mail: paragsharma8@rediffmail.com

DOI: $10.14260 /$ jemds $/ 2017 / 1450$
Changes acquired from the external environment reflect the baby's immature immune system or inability to adapt itself to its new environment.(2)

Both physiological and pathological skin changes during the early neonatal period cause a lot of concern amongst the parents. So, it becomes important to identify these changes correctly and allay the fears of the parents.

\section{Aims and Objectives}

1. To determine the relative incidence of various physiological and pathological skin changes during the early neonatal period.

2. To study the maternal and neonatal factors responsible for these changes.

3. To determine the time of appearance and disappearance of these skin changes.

4. The incidence of skin changes, its relation to maternal and neonatal factors and the time of their appearance and disappearance will be compared with earlier studies. 


\section{Review of Literature}

The skin of a neonate differs from that of an adult, both anatomically and physiologically. At birth the skin is covered by vernix caseosa.(3) After its removal, the skin looks intensely erythematous, smooth and soft in texture. Peripheral cyanosis is present at birth and is more marked on the palms, soles and around the mouth. After a few hours of birth, some babies develop harlequin colour change and cutis marmorata.(1) Sebaceous hyperplasia, milia, Epstein's pearls and Mongolian spots are present in a majority of neonates at birth or a few hours thereafter.(4)

The influence of maternal hormones on the foetus gives rise to numerous changes, which have been described as 'miniature puberty.' In the newborn females, the genitalia appear succulent with a large clitoris and mucoid vaginal discharge, which is followed by frank withdrawal bleeding. The male genitalia appear similarly large and well developed at birth. Both sexes show hypertrophy of the mammary glands and pigmentation of linea alba at birth.(5)

Hair loss over the scalp starts immediately after birth over the frontoparietal area producing an area of alopecia, that is analogous to adult male pattern alopecia. Physiological desquamation of the skin usually starts over the extremities by the $3^{\text {rd }}$ or $4^{\text {th }}$ day of life and then spreads to involve the entire body.(6) Physiological jaundice, which is manifested as yellowing of eyes and skin, is also seen during this period. Transient erythematous and pustular lesions in the form of erythema toxicum neonatorum, transient neonatal pustular melanosis and infantile acropustulosis usually starts on the $2^{\text {nd }}$ or $3^{\text {rd }}$ day of life.(7)

Infants born before $37^{\text {th }}$ week of gestation are considered as preterm infants. These infants are usually smaller in size, with thin, translucent skin and covered by lanugo hairs. The breast nodule, ear cartilage, genitalia and deep plantar creases are poorly developed. The postmature babies, i.e. infants born after 42 weeks of gestation are usually covered by greenish vernix caseosa. The skins of these babies are usually dry and parchment like.(8)

The pathological skin manifestations may be classified into infective skin disorders, non-infective skin dermatoses, genodermatoses and developmental skin defects.

Infective skin disorders can be caused by bacterial, fungal or viral infections. Bacterial infections include bullous impetigo, periporitis staphylogenes, staphylococcal scalded skin syndrome, ophthalmia neonatorum, necrotising fasciitis, omphalitis and breast abscess.(9) Fungal infections include oral and cutaneous candidiasis.(10) Viral infections like herpes simplex and varicella are rarely seen in neonates.

The non-infective skin dermatoses include miliaria, eczematous eruptions,(11) diseases of subcutaneous fat,(12) dermatoses acquired transplacentally and birth traumatic lesions.

The developmental skin defects include naevi, spina bifida and cleft lip. Vascular naevi like salmon patch,(13) port wine stain and strawberry haemangioma are seen at birth. Congenital melanocytic naevi, verrucous epidermal naevi and sebaceous naevi are occasionally seen at birth.(14)

Genodermatoses like ichthyosis, aplasia congenita cutis, epidermolysis bullosa, incontinentia pigmenti, erythropoietic porphyria and mastocytosis are also present.(15)

Cutaneous disorders of neonates were first described by Ballantyne (1895). Since then many reports have appeared in the literature.(4),(5),(8),(16),(17),(18),(19),(20) The factors influencing the pattern of cutaneous changes include climate, race, heredity, hygiene, maternal factors like mother's age and parity and neonatal factors like gestational age, sex and birth weight.

\section{MATERIALS AND METHODS}

1000 live-born babies delivered in labour room in 1 year period were selected for the present study. Babies who could remain in the hospital for at least 7 days were included in the study. This included babies delivered by CS, forceps and babies delivered vaginally, whose mothers remained in hospital for PPS. Mother's and baby's personal details were recorded. Cutaneous, General and Systemic examination of all babies were done. Babies were followed up daily till the next 7 days.

Laboratory examination like Gram staining, Wright's staining, Tzanck test, $\mathrm{KOH}$ preparation, culture and biopsy for histopathological examination were done whenever necessary.

\section{Sampling Method}

Convenience sampling technique.

\section{Sample Size}

1000 .

\section{Study Design}

Prospective observational study.

\section{Statistical Method}

Descriptive Statistics, Chi-square test, software used is SPSS 20.0 version.

\section{RESULTS}

\begin{tabular}{|c|c|c|c|c|}
\hline Birth & $\begin{array}{c}\text { Mother's } \\
\text { Number }\end{array}$ & Percentage & $\begin{array}{c}\text { Babies' } \\
\text { Number }\end{array}$ & Percentage \\
\hline Single & 964 & 98.3 & 964 & 96.4 \\
\hline Twins & 15 & 1.5 & 30 & 3 \\
\hline Triplets & 2 & 0.2 & 6 & 0.6 \\
\hline Total & $\mathbf{9 8 1}$ & $\mathbf{1 0 0}$ & $\mathbf{1 0 0 0}$ & $\mathbf{1 0 0}$ \\
\hline \multicolumn{6}{|c|}{ Table I. Demography of Mothers and Neonates } \\
\hline
\end{tabular}

Table I shows that 1000 neonates selected for study were born to 981 mothers, $98.3 \%$ of them delivered a single baby, $1.5 \%$ delivered twins and $0.2 \%$ delivered triplets.

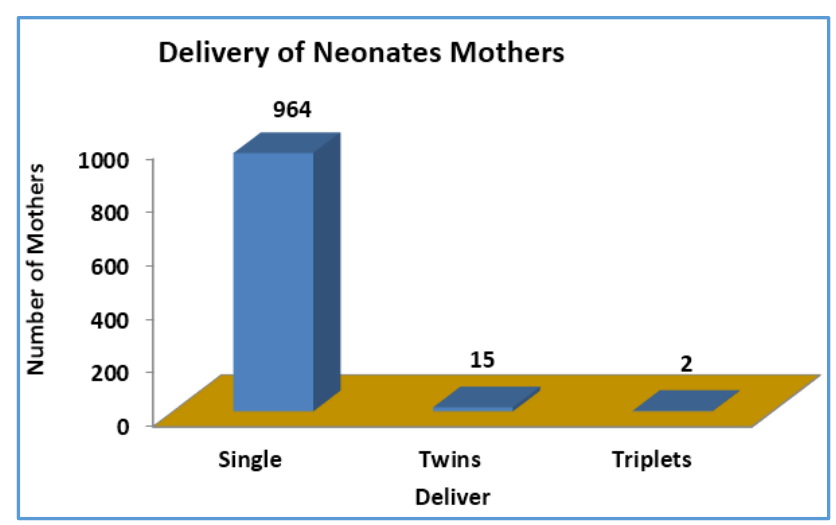




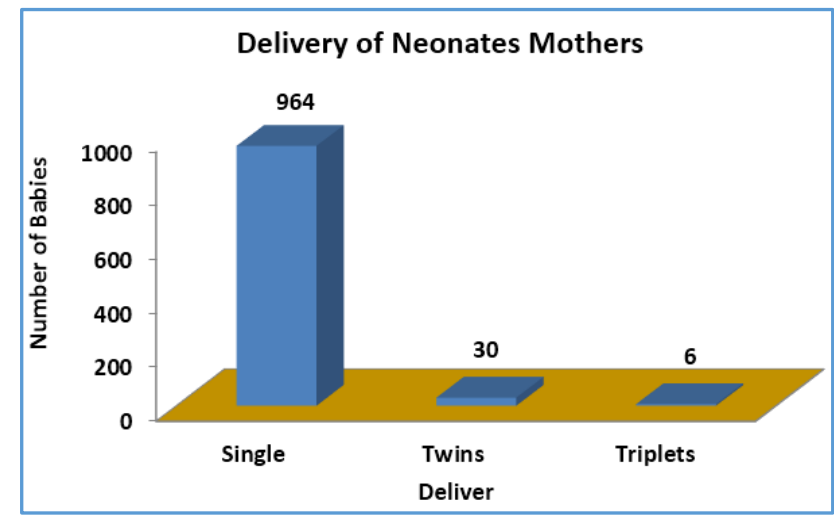

\begin{tabular}{|c|c|c|}
\hline Sex & Number & Percentage \\
\hline Male & 501 & 50.1 \\
\hline Female & 499 & 49.9 \\
\hline Total & $\mathbf{1 0 0 0}$ & $\mathbf{1 0 0}$ \\
\hline \multicolumn{2}{|r|}{ Table II. Sex Distribution of Neonates } \\
\hline
\end{tabular}

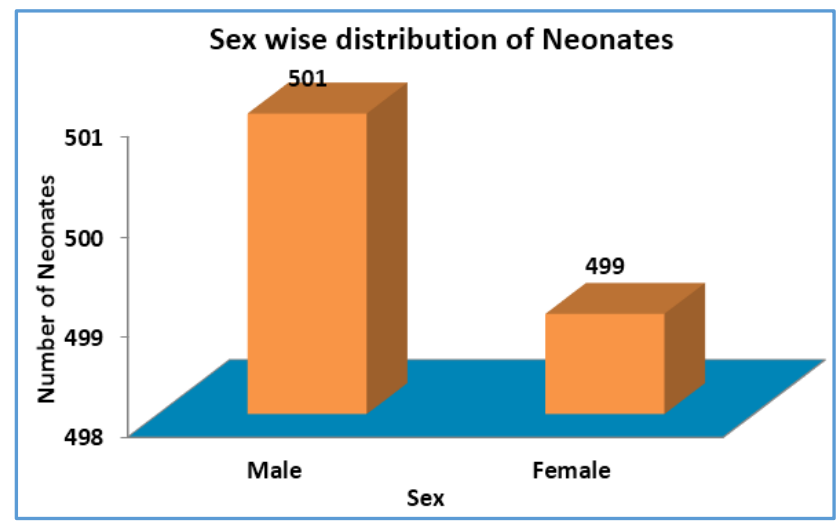

\begin{tabular}{|c|c|c|}
\hline Gestational Age & Number & Percentage \\
\hline Term & 789 & 78.9 \\
\hline Post-term & 110 & 11.0 \\
\hline Pre-term & 101 & 10.1 \\
\hline Total & $\mathbf{1 0 0 0}$ & $\mathbf{1 0 0}$ \\
\hline \multicolumn{2}{|c|}{ Table III. Gestational Age Distribution of Neonates } \\
\hline
\end{tabular}

Table II and Table III shows that out of 1000 neonates there were $50.1 \%$ males and $49.9 \%$ females, while $78.9 \%$ were term babies, $11 \%$ were post-term babies.

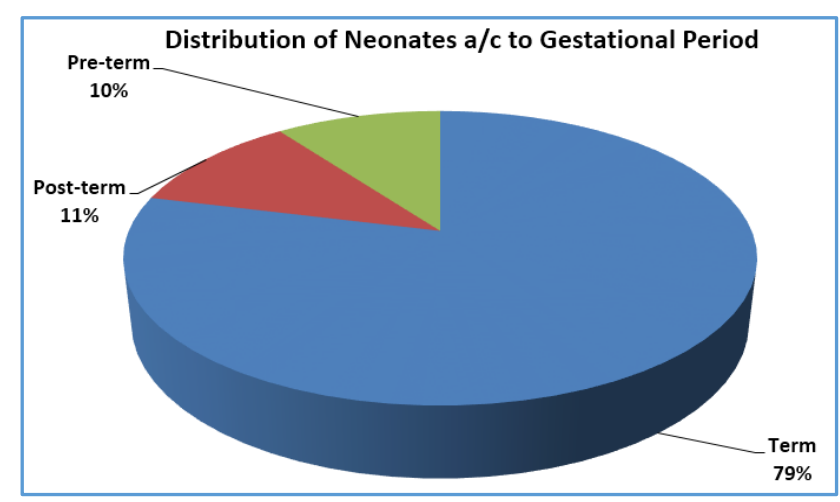

\begin{tabular}{|c|c|c|}
\hline $\begin{array}{c}\text { Birth Weight } \\
\text { in Grams }\end{array}$ & Number & Percentage \\
\hline$<2500$ & 204 & 20.4 \\
\hline$\geq 2500$ & 796 & 79.6 \\
\hline Total & $\mathbf{1 0 0 0}$ & $\mathbf{1 0 0}$ \\
\hline \multicolumn{2}{|c|}{ Table IV. Birth Weight of Neonates } \\
\hline
\end{tabular}

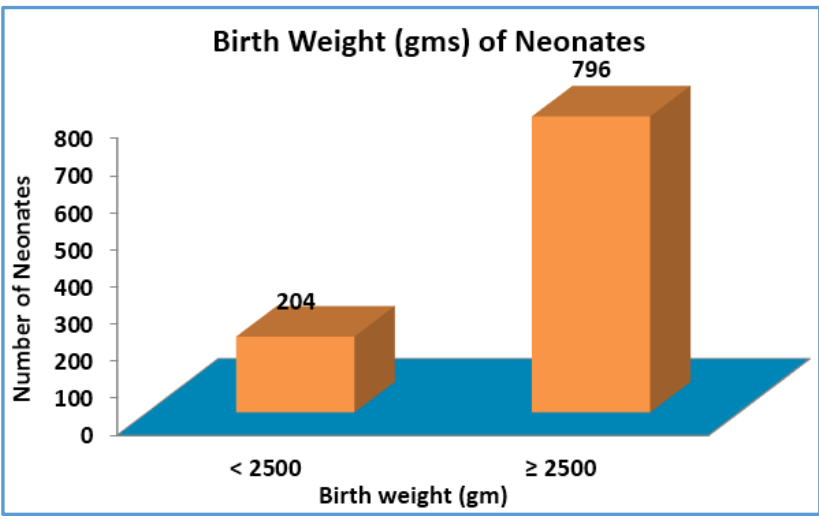

\begin{tabular}{|c|c|c|}
\hline Mode of Delivery & Number & Percentage \\
\hline Caesarean section & 681 & 68.1 \\
\hline Normal vaginal & 220 & 22 \\
\hline Forceps & 99 & 9.9 \\
\hline Total & $\mathbf{1 0 0 0}$ & $\mathbf{1 0 0}$ \\
\hline \multicolumn{2}{|r|}{ Table V. Mode of Delivery of Neonates } \\
\hline
\end{tabular}

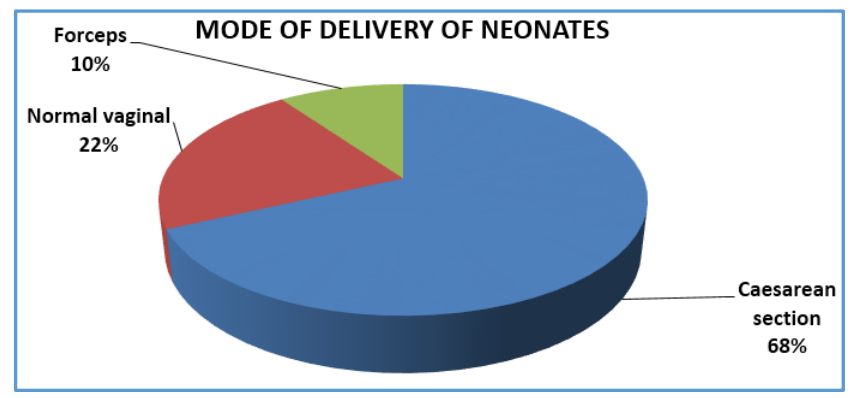

Table IV and Table $\mathrm{V}$ shows that out of 1000 neonates, $20.4 \%$ weighed below 2500 gms and $79.6 \%$ weighed above 2500 gms, while $68.1 \%$ neonates were delivered by caesarean section, $22 \%$ by normal vaginal delivery and $9.9 \%$ by forceps application.

\begin{tabular}{|c|c|c|c|}
\hline Sl. No. & Name of Skin Change & Number & Percentage \\
\hline 1 & Physiological scaling & 819 & 81.9 \\
\hline 2 & Recession of hair & 756 & 75.6 \\
\hline 3 & Mongolian spots & 698 & 69.8 \\
\hline 4 & Sebaceous hyperplasia & 633 & 63.3 \\
\hline 5 & Sparse hair & 586 & 58.6 \\
\hline 6 & Epstein's pearls & 560 & 56 \\
\hline 7 & Pigmentation of linea alba & 552 & 55.2 \\
\hline 8 & Milia & 491 & 49.1 \\
\hline 9 & $\begin{array}{l}\text { Erythema toxicum } \\
\text { neonatorum }\end{array}$ & 233 & 23.3 \\
\hline 10 & Dense hair & 190 & 19 \\
\hline 11 & Breast hypertrophy & 164 & 16.4 \\
\hline 12 & Physiological jaundice & 138 & 13.8 \\
\hline
\end{tabular}

Table VI shows that out of 1000 neonates, physiological desquamation of skin was the most common and physiological jaundice was the least common physiological skin changes. The incidence of physiological skin changes of our study was comparable with those of earlier workers. A 
high incidence of physiological desquamation, Mongolian spots, sebaceous hyperplasia and Epstein's pearls was seen in all the studies (Mishra 1988, Nobbay 1992, Nanda 1989). A low incidence of physiological jaundice and breast hypertrophy was noted in our study, which was similar to earlier reports (Nobbay 1992, Nanda 1989). However, a high incidence of recession of hair, sparse hair and pigmentation of linea alba was noted in our study which was not reported by earlier workers.

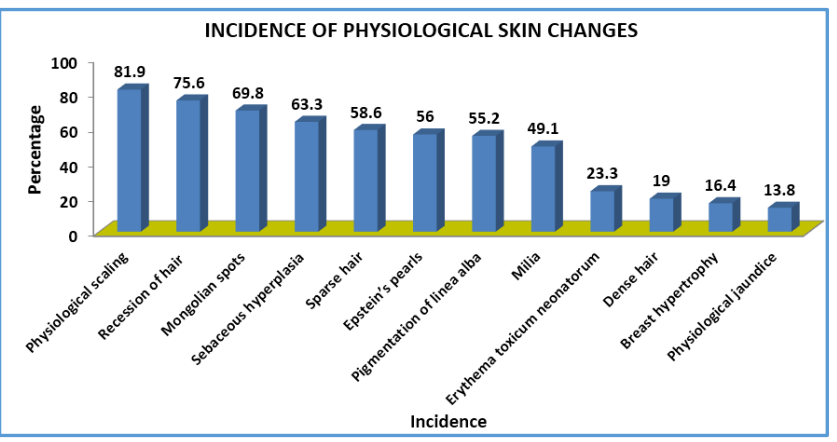

\begin{tabular}{|c|c|c|c|c|c|c|c|c|}
\hline Sl. No. & Name of Skin Change & Male & $\mathbf{\%}$ & Female & $\mathbf{\%}$ & Total & P-value* & Significant at 5\% Level \\
\hline 1 & Physiological scaling & 400 & 79.8 & 419 & 83.97 & 819 & 0.90 & No \\
\hline 2 & Recession of hair & 384 & 76.6 & 372 & 74.5 & 756 & 0.440 & No \\
\hline 3 & Mongolian spots & 338 & 67.5 & 360 & 72.1 & 698 & 0.107 & No \\
\hline 4 & Sebaceous hyperplasia & 358 & 71.5 & 275 & 55.1 & 633 & $<0.001$ & Yes \\
\hline 5 & Sparse hair & 354 & 70.7 & 232 & 46.5 & 586 & $<0.001$ & Yes \\
\hline 6 & Epstein's pearls & 279 & 55.7 & 281 & 56.3 & 560 & 0.842 & No \\
\hline 7 & Pigmentation of linea alba & 297 & 59.3 & 255 & 51.1 & 552 & 0.009 & Yes \\
\hline 8 & Milia & 221 & 44.1 & 270 & 54.1 & 491 & 0.002 & Yes \\
\hline 9 & $\begin{array}{c}\text { Erythema toxicum } \\
\text { neonatorum }\end{array}$ & 147 & 29.3 & 86 & 17.2 & 233 & $<0.001$ & Yes \\
\hline 10 & Dense hair & 79 & 15.8 & 111 & 22.2 & 190 & 0.009 & Yes \\
\hline 11 & Breast hypertrophy & 78 & 15.6 & 86 & 17.2 & 164 & 0.477 & No \\
\hline 12 & Physiological jaundice & 76 & 15.2 & 62 & 12.4 & 138 & 0.208 & No \\
\hline \multicolumn{7}{|c|}{ Table VII. Relation of Physiological Skin Changes to Neonate's Sex } \\
\hline
\end{tabular}

*Application of Chi-square Test.

Table VII shows that in the present study sebaceous hyperplasia, sparse hair, erythema toxicum neonatorum and dense hair showed a higher incidence among males, while milia and breast hypertrophy showed a higher incidence among female neonates. This was not reported by earlier workers.

\begin{tabular}{|c|c|c|c|c|c|c|c|c|c|c|}
\hline $\begin{array}{c}\text { Serial } \\
\text { No. }\end{array}$ & \begin{tabular}{|c|}
$\begin{array}{c}\text { Name of Skin } \\
\text { Change }\end{array}$ \\
\end{tabular} & $\begin{array}{c}\text { Term } \\
(n=789)\end{array}$ & Percent & $\begin{array}{c}\text { Pre-Term } \\
(\mathrm{n}=101)\end{array}$ & Percent & $\begin{array}{c}\text { Post-Term } \\
(\mathrm{n}=110)\end{array}$ & Percent & $\begin{array}{c}\text { Total } \\
(\mathrm{n}=\mathbf{1 0 0 0})\end{array}$ & P-value* & $\begin{array}{c}\text { Significant } \\
\text { at 5\% Level }\end{array}$ \\
\hline 1 & $\begin{array}{c}\text { Physiological } \\
\text { scaling }\end{array}$ & 686 & 86.9 & 25 & 24.8 & 108 & 98.2 & 819 & $<0.001$ & Yes \\
\hline 2 & $\begin{array}{c}\text { Recession of } \\
\text { hair }\end{array}$ & 602 & 76.3 & 56 & 55.4 & 98 & 89.1 & 756 & $<0.001$ & Yes \\
\hline 3 & $\begin{array}{l}\text { Mongolian } \\
\text { spots }\end{array}$ & 556 & 70.5 & 51 & 50.5 & 91 & 82.7 & 698 & $<0.001$ & Yes \\
\hline 4 & $\begin{array}{c}\text { Sebaceous } \\
\text { hyperplasia }\end{array}$ & 489 & 61.98 & 83 & 82.2 & 61 & 55.5 & 633 & $<0.001$ & Yes \\
\hline 5 & Sparse hair & 459 & 58.2 & 52 & 51.5 & 75 & 68.2 & 586 & 0.042 & Yes \\
\hline 6 & $\begin{array}{l}\text { Epstein's } \\
\text { pearls }\end{array}$ & 460 & 58.3 & 51 & 50.5 & 49 & 44.5 & 560 & 0.012 & Yes \\
\hline 7 & $\begin{array}{c}\text { Pigmentation } \\
\text { of linea alba }\end{array}$ & 409 & 51.8 & 73 & 72.3 & 70 & 63.6 & 552 & $<0.001$ & Yes \\
\hline 8 & Milia & 422 & 53.5 & 26 & 25.7 & 43 & 39.1 & 491 & $<0.001$ & Yes \\
\hline 9 & $\begin{array}{c}\text { Erythema } \\
\text { toxicum } \\
\text { neonatorum }\end{array}$ & 180 & 22.8 & 14 & 13.9 & 39 & 35.6 & 233 & 0.001 & Yes \\
\hline 10 & Dense hair & 163 & 20.7 & 21 & 20.8 & 6 & 5.5 & 190 & 0.001 & Yes \\
\hline 11 & $\begin{array}{c}\text { Breast } \\
\text { hypertrophy }\end{array}$ & 143 & 18.1 & 5 & 4.95 & 16 & 14.5 & 164 & 0.003 & Yes \\
\hline 12 & $\begin{array}{c}\text { Physiological } \\
\text { jaundice }\end{array}$ & 101 & 12.8 & 24 & 23.8 & 13 & 11.8 & 138 & 0.009 & Yes \\
\hline
\end{tabular}

*Application of Chi-square Test.

Table VIII shows that Preterm babies showed a higher incidence of sebaceous hyperplasia (Nanda 1989) and physiological jaundice (Dutta 1989) and a lower incidence of physiological scaling, milia (Mishra 1985), erythema toxicum neonatorum (LaVoo 1994) and breast hypertrophy (Rudoy 1975), which corresponds with the earlier studies. Preterm babies showed a higher incidence of pigmentation of linea alba and a lower incidence of hair recession and Mongolian spots which was not reported earlier. Post-term babies showed a higher incidence of sparse hair and physiological scaling (Wagner 1995), which was seen in earlier studies. Term babies showed a significantly higher incidence of Epstein's pearls, which was not reported earlier. 


\begin{tabular}{|c|c|c|c|c|c|c|c|c|}
\hline $\begin{array}{c}\text { Serial } \\
\text { No. }\end{array}$ & Name of Skin Change & $\mathbf{< 2 5 0 0} \mathbf{g}$ & Percent & $\mathbf{2} \mathbf{2 5 0 0} \mathbf{g}$ & Percent & Total & P-value* & $\begin{array}{c}\text { Significant at } \\
\mathbf{5 \%} \text { Level }\end{array}$ \\
\hline 1 & Physiological scaling & 140 & 68.6 & 679 & 85.3 & 819 & $<0.001$ & Yes \\
\hline 2 & Recession of hair & 145 & 71.1 & 611 & 76.6 & 756 & 0.92 & No \\
\hline 3 & Mongolian spots & 154 & 75.5 & 544 & 68.3 & 698 & 0.047 & Yes \\
\hline 4 & Sebaceous hyperplasia & 153 & 75 & 480 & 60.3 & 633 & $<0.001$ & Yes \\
\hline 5 & Sparse hair & 133 & 65.2 & 453 & 56.9 & 586 & 0.032 & Yes \\
\hline 6 & Epstein's pearls & 126 & 61.8 & 434 & 54.5 & 560 & 0.063 & No \\
\hline 7 & Pigmentation of linea alba & 107 & 52.5 & 445 & 55.9 & 552 & 0.376 & No \\
\hline 8 & Milia & 101 & 49.5 & 390 & 49 & 491 & 0.896 & No \\
\hline 9 & $\begin{array}{c}\text { Erythema toxicum } \\
\text { neonatorum }\end{array}$ & 23 & 11.3 & 210 & 26.4 & 233 & $<0.001$ & Yes \\
\hline 10 & Dense hair & 38 & 18.6 & 152 & 19.1 & 190 & 0.879 & No \\
\hline 11 & Breast hypertrophy & 17 & 8.3 & 147 & 18.5 & 164 & $<0.001$ & Yes \\
\hline 12 & Physiological jaundice & 34 & 16.7 & 104 & 13.1 & 138 & 0.183 & No \\
\hline \multicolumn{2}{r|}{ Table IX. Relation of Physiological Skin Changes to Neonates Birth Weight } \\
\end{tabular}

*Application of Chi-square Test.

Table IX shows that Neonates weighing above $2.5 \mathrm{~kg}$ showed a higher incidence of erythema toxicum neonatorum (Rohr 1975 , Kulkarni 1996), which was also reported in earlier studies. In our study neonates weighing below $2.5 \mathrm{~kg}$ showed a higher incidence of sebaceous hyperplasia, while neonates weighing above $2.5 \mathrm{~kg}$ showed a higher incidence of physiological scaling and breast hypertrophy which was not reported earlier. Kulkarni (1996) reported a higher incidence of Epstein's pearls in neonates weighing above $2.5 \mathrm{~kg}$, which was not observed in our study.

\begin{tabular}{|c|c|c|c|}
\hline Serial No. & Name of Skin Disorder & Number of Neonates & Percentage \\
\hline 1 & Ophthalmia neonatorum & 233 & 23.3 \\
\hline 2 & Bullous impetigo & 89 & 8.9 \\
\hline 3 & Oral candidiasis & 26 & 2.6 \\
\hline 4 & Periporitis staphylogenes & 17 & 1.7 \\
\hline 5 & Breast abscess & 1 & 0.1 \\
\hline \multicolumn{2}{|c|}{ Table X. Incidence of Infectious Skin Disorders } \\
\hline
\end{tabular}

Table X shows that out of 1000 neonates, ophthalmia neonatorum (23.3\%) was the most common and breast abscess (0.1\%) was the least common infectious skin disorders. The high incidence of Bullous impetigo, Oral candidiasis and Breast abscess of the present study was comparable with those of earlier workers (Rudoy 1975, Nanda 1989, Nobbay 1992). The high incidence of Ophthalmia neonatorum (23.3\%) and Periporitis staphylogenes (1.7\%) was not mentioned previously.

\begin{tabular}{|c|c|c|c|c|c|c|c|c|c|c|}
\hline $\begin{array}{c}\text { Serial } \\
\text { No. }\end{array}$ & $\begin{array}{c}\text { Name of Skin } \\
\text { Change }\end{array}$ & $\begin{array}{c}\text { Term } \\
(\mathbf{n = 7 8 9})\end{array}$ & Percent & $\begin{array}{c}\text { Pre-Term } \\
(\mathbf{n = 1 0 1})\end{array}$ & Percent & $\begin{array}{c}\text { Post-Term } \\
(\mathbf{n = 1 1 0})\end{array}$ & Percent & $\begin{array}{c}\text { Total } \\
(\mathbf{n = 1 0 0 0 )}\end{array}$ & $\begin{array}{c}\text { P-value** } \\
\text { Significant } \\
\text { at 5\% Level }\end{array}$ \\
\hline 1 & $\begin{array}{c}\text { Ophthalmia } \\
\text { neonatorum }\end{array}$ & 184 & 23.3 & 25 & 24.8 & 24 & 21.8 & 233 & 0.880 & No \\
\hline 2 & $\begin{array}{c}\text { Bullous } \\
\text { impetigo }\end{array}$ & 80 & 10.1 & 3 & 2.97 & 6 & 6 & 89 & 0.024 & Yes \\
\hline 3 & $\begin{array}{c}\text { Oral } \\
\text { candidiasis }\end{array}$ & 8 & 1 & 6 & 5.9 & 12 & 12 & 26 & $<0.001$ & Yes \\
\hline 4 & $\begin{array}{c}\text { Periporitis } \\
\text { staphylogenes }\end{array}$ & 13 & 1.6 & 3 & 2.97 & 1 & 1 & 17 & 0.497 & No \\
\hline 5 & Breast abscess & 1 & 0.1 & 0 & 0 & 0 & 0 & 1 & 0.875 & No \\
\hline \multicolumn{7}{|c|}{ Table XI. Relation of Infectious Skin Disorders to Gestational Age of Neonate } \\
\hline
\end{tabular}

*Application of Chi-square Test.

Table XI shows that term neonates showed a significantly higher incidence of bullous impetigo, while post-term neonates showed a significantly higher incidence of oral candidiasis. However, infectious skin disorders of neonates did not vary significantly with neonates' sex and birth weight or with mother's age and parity.

\begin{tabular}{|c|c|c|c|}
\hline $\begin{array}{c}\text { Serial } \\
\text { No. }\end{array}$ & Name of Skin Disorder & Number of Neonates & Percentage \\
\hline 1 & Miliaria crystallina & 353 & 95.3 \\
\hline 2 & Scalp ecchymosis & 71 & 7.1 \\
\hline 3 & Perianal dermatitis & 56 & 5.6 \\
\hline 4 & Miliaria rubra & 24 & 2.4 \\
\hline 5 & Caput succedaneum & 9 & 0.9 \\
\hline 6 & Sclerema neonatorum & 1 & 0.1 \\
\hline \multicolumn{2}{|c|}{ Table XII. Incidence of Non-Infectious Skin Disorders } \\
\hline
\end{tabular}


Table XII shows that out of 1000 neonates, miliaria crystallina (35.3\%) was the most common and sclerema neonatorum $(0.1 \%)$ was the least common non-infectious skin disorders. The high incidence of Scalp ecchymosis, Perianal dermatitis, Miliaria rubra and Sclerema neonatorum of our study corresponded with the earlier studies (Nanda 1989, Rivers 1990, Nobbay 1992). The incidence of Miliaria crystallina was higher and that of Caput succedaneum lower than previous studies (Nanda 1989), probably because our study was done in a hot environment and forceps delivery was less.

\begin{tabular}{|c|c|c|c|c|c|c|c|c|c|c|}
\hline $\begin{array}{c}\text { Serial } \\
\text { No. }\end{array}$ & $\begin{array}{c}\text { Name of } \\
\text { Skin Change }\end{array}$ & $\begin{array}{c}\text { Term } \\
(\mathbf{n = 7 8 9})\end{array}$ & $\mathbf{\%}$ & $\begin{array}{c}\text { Pre-Term } \\
(\mathbf{n = 1 0 1})\end{array}$ & $\mathbf{\%}$ & $\begin{array}{c}\text { Post-Term } \\
(\mathbf{n = 1 1 0})\end{array}$ & $\mathbf{\%}$ & $\begin{array}{c}\text { Total } \\
(\mathbf{n = 1 0 0 0 )}\end{array}$ & P-value* & $\begin{array}{c}\text { Significant at } \\
\mathbf{5 \%} \text { Level }\end{array}$ \\
\hline 1 & Miliaria crystallina & 288 & 36.5 & 26 & 25.7 & 39 & 35.5 & 353 & 0.103 & No \\
\hline 2 & Scalp ecchymosis & 51 & 6.5 & 3 & 3 & 17 & 15.5 & 71 & 0.001 & Yes \\
\hline 3 & Perianal dermatitis & 30 & 3.8 & 18 & 17.8 & 8 & 7.3 & 56 & $<0.001$ & Yes \\
\hline 4 & Miliaria rubra & 15 & 1.9 & 0 & 0 & 9 & 8.2 & 24 & $<0.001$ & Yes \\
\hline 5 & Caput succedaneum & 6 & 0.8 & 0 & 0 & 3 & 2.7 & 9 & 0.660 & No \\
\hline 6 & Tclerema neonatorum & 0 & 0 & 1 & 0.1 & 0 & 0 & 1 & 0.012 & Yes \\
\hline \multicolumn{10}{|c|}{ Table XIII. Relation of Non-Infectious Disorders to Gestational Age of Neonate } \\
\hline
\end{tabular}

Table XIII shows that preterm neonates showed a significantly higher incidence of perianal dermatitis, probably due to increased skin fragility. Preterm neonates showed a significantly lower incidence of miliaria crystallina. Post-term neonates showed a significantly higher incidence of scalp ecchymosis.

\begin{tabular}{|c|c|c|c|}
\hline $\begin{array}{l}\text { Sl. } \\
\text { No. }\end{array}$ & $\begin{array}{l}\text { Name of Skin } \\
\text { Disorder }\end{array}$ & $\begin{array}{c}\text { Number of } \\
\text { Neonates }\end{array}$ & $\%$ \\
\hline 1 & Salmon patch & 190 & 19.0 \\
\hline 2 & Café-au-lait macules & 5 & 0.5 \\
\hline 3 & $\begin{array}{c}\text { Congenital melanocytic } \\
\text { nevus }\end{array}$ & 3 & 0.3 \\
\hline 4 & Port wine stain & 2 & 0.2 \\
\hline 5 & Cleft lip & 2 & 0.2 \\
\hline 6 & Epidermal nevus & 1 & 0.1 \\
\hline 7 & Skin tags & 1 & 0.1 \\
\hline 8 & Spina bifida & 1 & 0.1 \\
\hline \multicolumn{4}{|c|}{ Table XIV. Incidence of Developmental Defects } \\
\hline
\end{tabular}

Table XIV shows that out of 1000 neonates, Salmon patch $(19 \%)$ was the most common and Spina bifida $(0.1 \%)$ was the least common developmental defects. The incidence of Salmon patch in our study was significantly lower than the studies carried out by earlier workers (Dickson 1979, Nanda 1989, Rivers 1990). In our study a significantly higher incidence of Salmon patch was seen among males, neonates weighing below $2.5 \mathrm{~kg}$ and in primiparas which was not reported earlier. In the present study, the incidence of Congenital melanocytic nevus (Mayerhofer 1927), Port wine stain (Jacobs 1976), Cleft lip, Epidermal nevus (Rivers 1990), Skin tags and Spina bifida (Nanda 1989) was comparable with the findings of earlier workers.

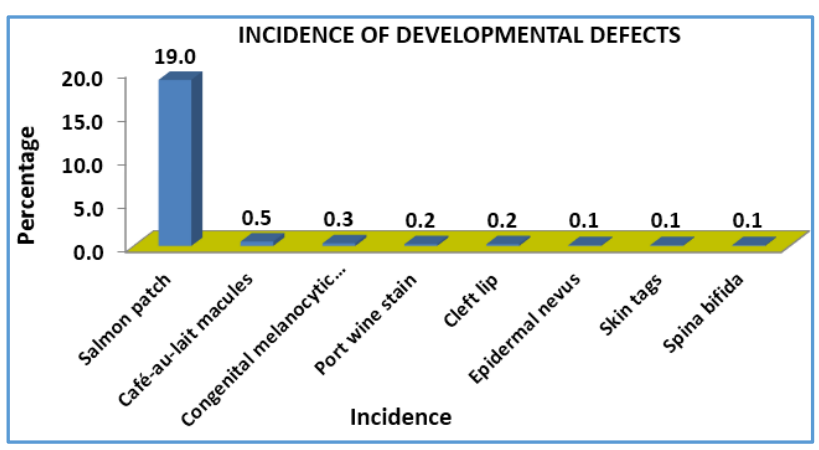

\begin{tabular}{|c|c|c|c|c|c|c|c|c|}
\hline $\begin{array}{c}\text { Sl. } \\
\text { No. }\end{array}$ & $\begin{array}{c}\text { Name of Skin } \\
\text { Changes }\end{array}$ & $\begin{array}{c}\text { Birth } \\
\text { and } \\
\text { 1st }^{\text {Day }}\end{array}$ & $\begin{array}{l}\text { 2nd } \\
\text { Day }\end{array}$ & $\begin{array}{l}3 \text { rd } \\
\text { Day }\end{array}$ & $\begin{array}{l}4^{\text {th }} \\
\text { Day }\end{array}$ & $\begin{array}{c}5^{\text {th }} \\
\text { Day }\end{array}$ & $\begin{array}{l}6^{\text {th }} \\
\text { Day }\end{array}$ & $\begin{array}{c}7^{\text {th }} \\
\text { Day }\end{array}$ \\
\hline 1 & $\begin{array}{l}\text { Physiological } \\
\text { scaling }\end{array}$ & $*$ & * & 145 & 127 & 256 & 182 & 109 \\
\hline 2 & $\begin{array}{l}\text { Recession of } \\
\text { hair }\end{array}$ & 671 & 37 & 28 & 20 & * & * & * \\
\hline 3 & $\begin{array}{l}\text { Mongolian } \\
\text { spots }\end{array}$ & 464 & 234 & * & * & * & * & * \\
\hline 4 & $\begin{array}{c}\text { Sebaceous } \\
\text { hyperplasia }\end{array}$ & 568 & 37 & 28 & * & * & * & * \\
\hline 5 & Sparse hair & 441 & 87 & 19 & 39 & $*$ & $*$ & $*$ \\
\hline 6 & Epstein's pearls & 540 & 9 & 11 & $*$ & * & $*$ & $*$ \\
\hline 7 & $\begin{array}{c}\text { Pigmentation of } \\
\text { linea alba }\end{array}$ & 394 & 73 & 85 & * & $*$ & * & * \\
\hline 8 & Milia & 378 & 40 & 73 & * & $*$ & * & $*$ \\
\hline 9 & $\begin{array}{l}\text { Erythema } \\
\text { toxicum } \\
\text { neonatorum }\end{array}$ & 29 & 107 & 68 & 29 & $*$ & $*$ & * \\
\hline 10 & Dense hair & 145 & 18 & 27 & * & $*$ & * & $*$ \\
\hline 11 & $\begin{array}{c}\text { Breast } \\
\text { hypertrophy }\end{array}$ & 86 & 52 & 26 & * & * & * & * \\
\hline 12 & $\begin{array}{l}\text { Physiological } \\
\text { jaundice }\end{array}$ & $*$ & $*$ & 26 & 95 & 17 & $*$ & * \\
\hline 13 & Total & 3716 & 694 & 536 & 310 & 273 & 182 & 109 \\
\hline 14 & Percentage & 63.9 & 11.9 & 9.2 & 5.3 & 4.7 & 3.1 & \begin{tabular}{|l|}
1.9 \\
\end{tabular} \\
\hline \multicolumn{9}{|c|}{$\begin{array}{c}\text { Table XV. Days of Appearance of Physiological Skin } \\
\text { Changes }\end{array}$} \\
\hline
\end{tabular}

Table XV shows that out of 5820 physiological skin changes seen among 1000 neonates, maximum number (3716) appeared on the first day and minimum number (109) appeared on the seventh day of life. Most of the lesions of Mongolian spots, recession of hair, sebaceous hyperplasia, sparse hair, Epstein's pearls, pigmentation of linea alba, milia, dense hair and breast hypertrophy appeared on the first day. Majority of the lesions of erythema toxicum neonatorum, physiological jaundice and physiological scaling appeared on $2^{\text {nd }}, 4^{\text {th }}$ and $5^{\text {th }}$ day respectively. In our study, physiological scaling appeared from $3^{\text {rd }}$ day onwards reaching a peak on $5^{\text {th }}$ day, which was in contrast to other studies where it appeared on $1^{\text {st }}$ day (Hodgman 1971, Atherton 1992). The hot weather condition prevailing in our area of study could be the result of 
this discrepancy. $67 \%$ of Mongolian spots were seen on the $1^{\text {st }}$ day of our study, which was significantly lower than earlier studies (Dickson 1979). Most of our babies were of dark skin, making it difficult to observe Mongolian spots at birth. As the Mongolian spots darkened after birth, a significantly higher incidence was observed on the $2^{\text {nd }}$ day.

The day of appearance of hair recession, sebaceous hyperplasia, sparse hair, milia, dense hair, rash of erythema toxicum neonatorum and physiological jaundice were similar to earlier studies (Steigleder 1963, Nanda 1989, Rivers 1990). 94\% Epstein's pearls, $71.4 \%$ pigmentation of linea alba and $52 \%$ breast hypertrophy were observed on the $1^{\text {st }}$ day, which was not reported by earlier workers.

\begin{tabular}{|c|c|c|c|c|c|c|c|c|}
\hline \begin{tabular}{l|} 
Sl. \\
No.
\end{tabular} & $\begin{array}{c}\text { Name of Skin } \\
\text { Disorders }\end{array}$ & \begin{tabular}{|l} 
Birth \\
and \\
1st \\
Day
\end{tabular} & $\begin{array}{l}2^{\text {nd }} \\
\text { Day }\end{array}$ & $\begin{array}{l}3^{\text {rd }} \\
\text { Day }\end{array}$ & $\begin{array}{c}4^{\text {th }} \\
\text { Day }\end{array}$ & $\begin{array}{c}5^{\text {th }} \\
\text { Day }\end{array}$ & $\begin{array}{c}6^{\text {th }} \\
\text { Day }\end{array}$ & $\begin{array}{l}7^{\text {th }} \\
\text { Day }\end{array}$ \\
\hline 1 & $\begin{array}{c}\text { Miliaria } \\
\text { crystallina }\end{array}$ & 91 & 109 & 38 & 24 & 24 & 38 & 29 \\
\hline 2 & $\begin{array}{l}\text { Ophthalmia } \\
\text { neonatorum }\end{array}$ & 36 & 62 & 63 & 54 & 9 & $*$ & 9 \\
\hline 3 & Bullous impetigo & * & * & * & 39 & 25 & 6 & 19 \\
\hline 4 & Scalp ecchymosis & 71 & $*$ & $*$ & $*$ & $*$ & * & $*$ \\
\hline 5 & $\begin{array}{c}\text { Perianal } \\
\text { dermatitis }\end{array}$ & $*$ & $*$ & $*$ & 10 & 11 & 14 & 21 \\
\hline 6 & Miliaria rubra & $*$ & 17 & 13 & 4 & * & $*$ & $*$ \\
\hline 7 & Oral candidiasis & $*$ & $*$ & $*$ & 10 & 3 & 6 & 7 \\
\hline 8 & $\begin{array}{c}\text { Periporitis } \\
\text { staphylogenes }\end{array}$ & $*$ & $*$ & 4 & 13 & $*$ & $*$ & $*$ \\
\hline 9 & $\begin{array}{c}\text { Caput } \\
\text { succedaneum }\end{array}$ & 9 & * & $*$ & $*$ & $*$ & $*$ & $*$ \\
\hline 10 & Breast abscess & $*$ & * & * & * & $*$ & * & 1 \\
\hline 11 & $\begin{array}{c}\text { Sclerema } \\
\text { neonatorum }\end{array}$ & $*$ & 1 & $*$ & $*$ & $*$ & $*$ & $*$ \\
\hline 12 & Total & 207 & 189 & 118 & 154 & 72 & 64 & 86 \\
\hline 13 & Percentage & 23.2 & 21.2 & 13.3 & & 8.1 & 7.2 & 9.7 \\
\hline \multicolumn{9}{|c|}{$\begin{array}{c}\text { Table XVI. Day of Appearance of Pathological Skin } \\
\text { Manifestations }\end{array}$} \\
\hline
\end{tabular}

Table XVI shows that out of 890 infectious and noninfectious skin changes seen among 1000 babies, the lesions appeared with an equal frequency throughout the 7 days. Lesions of malaria crystalline appeared on all 7 days of life. Ophthalmia neonatorum was more frequently seen on first 4 days of life. Lesions of bullous impetigo, oral candidiasis and perianal dermatitis appeared between $4^{\text {th }}$ to $7^{\text {th }}$ day of life. Lesions of Miliaria rubra appeared on $2^{\text {nd }}$ to $4^{\text {th }}$ day, while lesions of Periporitis staphylogenes appeared on $3^{\text {rd }}$ to $4^{\text {th }}$ day of life. Only one case of breast abscess was seen and it appeared on the $7^{\text {th }}$ day, while one case of sclerema neonatorum was seen on $2^{\text {nd }}$ day.

The day of appearance of Miliaria crystalline, Bullous impetigo, Oral candidiasis, Breast abscess and Sclerema neonatorum was similar to earlier studies (Smith 1965, Hodgman 1971, Rudoy 1975, Atherton 1992, Wagner 1995). Pratt et al (1951) reported that the lesions of Perianal dermatitis appeared on the $1^{\text {st }}$ day, while we found that it appeared between $4^{\text {th }}-7^{\text {th }}$ day. We found that the lesions of Miliaria rubra appeared between $2^{\text {nd }}$ to $4^{\text {th }}$ day and those of Ophthalmia neonatorum appeared on the first 4 days of life which was not reported earlier.

\begin{tabular}{|c|c|c|c|c|c|}
\hline Skin Changes & & \begin{tabular}{|c|} 
No. of \\
Neonates
\end{tabular} & $\%$ & \begin{tabular}{|c|} 
No. of \\
Neonates
\end{tabular} & $\%$ \\
\hline Physiological & & & & 5820 & $84.3 \%$ \\
\hline \multirow[t]{3}{*}{ Pathological } & Infectious & 366 & $5.3 \%$ & & \\
\hline & $\begin{array}{c}\text { Non- } \\
\text { Infectious }\end{array}$ & 514 & $7.4 \%$ & & \\
\hline & $\begin{array}{c}\text { Develop- } \\
\text { mental }\end{array}$ & 205 & $3 \%$ & & \\
\hline $\begin{array}{c}\text { Total } \\
\text { Pathological }\end{array}$ & & & & 1085 & $15.7 \%$ \\
\hline $\begin{array}{c}\text { Total Skin } \\
\text { Changes }\end{array}$ & & & & 6905 & $100 \%$ \\
\hline
\end{tabular}

Table XVII. Total Skin Changes in 1000 Neonates

Table XVII shows that 6905 skin changes were observed in 1000 neonates at an average of 6.9 lesions per neonate. There were 5820 (84.3\%) physiological skin changes and 1085 (15.7\%) pathological skin changes. Pathological skin changes include 366 (5.3\%) infectious, 514 (7.4\%) noninfectious and 205 (3\%) developmental defects.

\begin{tabular}{|c|c|c|}
\hline $\begin{array}{c}\text { Skin Lesions requiring No } \\
\text { Treatment }\end{array}$ & $\begin{array}{c}\text { Number of } \\
\text { Neonates }\end{array}$ & $\%$ \\
\hline Physiological skin changes & 5820 & $90.9 \%$ \\
\hline $\begin{array}{l}\text { Developmental defects other than } \\
\text { spina bifida and cleft lip }\end{array}$ & 202 & $3.2 \%$ \\
\hline Miliaria crystallina & 353 & $5.5 \%$ \\
\hline Miliaria rubra & 24 & $0.4 \%$ \\
\hline Total & 6399 & $100 \%$ \\
\hline
\end{tabular}

Table XVIII shows that out of 6905 skin changes, 6399 skin lesions (92.7\%) required no treatment. Out of 6399 skin lesions, 5499 skin lesions (83\%) disappeared within 3 - 4 days. Mongolian spots (698) and lesions of Developmental defects (202) remained even after one week. Most of Mongolian spots disappeared between 6 to 12 months. Salmon patch becomes lighter within the first week and usually disappears by 1 month. Skin tags also disappear spontaneously or it can be removed. Café-au-lait macules, Port wine stain, CMN and Epidermal nevus remains for life and can be treated for cosmetic purpose.

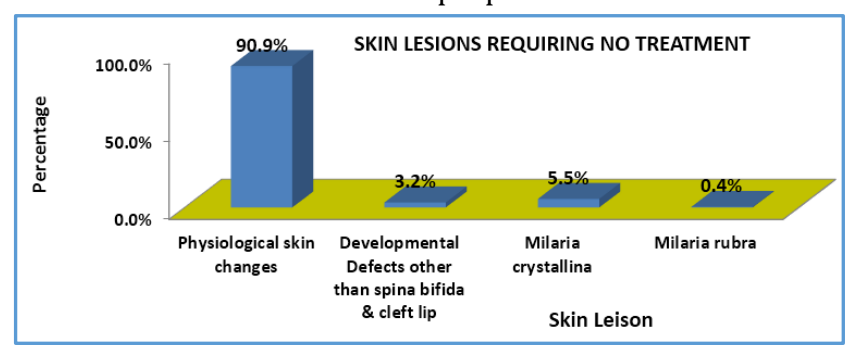

\begin{tabular}{|c|c|c|}
\hline $\begin{array}{c}\text { Skin Lesions requiring } \\
\text { Treatment }\end{array}$ & $\begin{array}{c}\text { Number of } \\
\text { Neonates }\end{array}$ & Percentage \\
\hline Ophthalmia neonatorum & 233 & $46.0 \%$ \\
\hline Bullous impetigo & 89 & $17.6 \%$ \\
\hline Oral candidiasis & 26 & $5.1 \%$ \\
\hline Periporitis staphylogenes & 17 & $3.4 \%$ \\
\hline Breast abscess & 1 & $0.2 \%$ \\
\hline Scalp ecchymosis & 71 & $14.0 \%$ \\
\hline Perianal dermatitis & 56 & $11.1 \%$ \\
\hline Caput succedaneum & 9 & $1.8 \%$ \\
\hline Cleft lip & 2 & $0.4 \%$ \\
\hline Sclerema neonatorum & 1 & $0.2 \%$ \\
\hline Spina bifida & 1 & $0.2 \%$ \\
\hline Total & $\mathbf{5 0 6}$ & $\mathbf{1 0 0} \%$ \\
\hline Table XIX. Skin Lesions requiring Treatment \\
\hline \multicolumn{2}{|c}{}
\end{tabular}


Table XIX shows that out of 6905 skin changes, 506 $(7.3 \%)$ lesions required some treatment. Cleft lip is treated surgically. Spina bifida should be surgically operated immediately after birth. Amongst the skin lesions which required treatment, only baby with sclerema neonatorum was treated in NICU. Some of the Babies with Caput succedaneum were irritable. So babies with Caput succedaneum were kept under strict observation. Rest of the babies recovered within 5 days after topical treatment.

\section{DISCUSSION}

Physiological skin changes were found more commonly than pathological skin changes and the ratio between physiological to pathological skin changes was 5.8: 1.1. Physiological skin changes varied significantly with neonatal and maternal factors, while most of the pathological skin changes did not vary with the above factors. The incidence of physiological and pathological skin changes in our study, their relation to maternal and neonatal factors and the time of their appearance and disappearance corresponded to that of earlier studies.

Most of the pathological skin disorders were either acquired from the external environment or transmitted genetically from the parents. Thus, maintenance of good hygiene with appropriate prenatal investigations will prevent the occurrence of the pathological skin changes. In our study, it was seen that only 2 out of 6905 skin lesions required immediate intervention. So, we can assure the parents that all babies will develop some or the other skin lesions during their early neonatal period. Most of them will disappear spontaneously or with minimal treatment. Rarely immediate intervention is required. Parents should observe whether their babies are irritable, constantly crying, not drinking milk or having cold extremities and bring it to doctor's notice. They should not unnecessarily observe and fiddle with the skin lesions. Nothing should be applied or fed to the baby.

\section{CONCLUSION}

Physiological skin changes were found more commonly than pathological skin changes and the ratio between physiological to pathological skin changes was 5.8: 1.1. Physiological skin changes varied significantly with neonatal and maternal factors, while most of the pathological skin changes did not vary with the above factors. The parents can be assured that their babies will develop some skin lesions during their early neonatal period. Most of them will disappear spontaneously or with minimal treatment. Rarely immediate intervention is required.

\section{REFERENCES}

[1] Paige DG, Genmeryar AR, Cant AJ. The Neonate. In: Burns T, Breathnach S, Cox N, et al. eds. Rook's textbook of dermatology. $8^{\text {th }}$ edn. New York: Wiley Blackwell Publication 2010;17:17.1-17.10.

[2] Kulkarni ML, Singh R. Normal variants of skin in neonates. Ind J Dermatol Venereol Leprol 1996;62(2):83-6.
[3] Downing DT, Strauss JS. Synthesis and composition of surface lipids of human skin. J Invest Dermatol 1974;1: 437-42.

[4] Nanda A, Kaur S, Bhakoo ON, et al. Survey of cutaneous lesions in Indian newborns. Pediatr Dermatol 1989;6(1):39-42.

[5] Nobbay B, Chakravarty N. Cutaneous manifestations in the newborn. Ind J Dermatol Venereol Leprol 1992;58(2):69-72.

[6] Griffths AD. Skin desquamation in the newborn. Bio Neonate 1966;10(3):127-39.

[7] Mayerhofer E, Lypolt-Kranjnovic M. Das erythema neonatorum toxicum Leiner ("Erythema papulatum" der alte aerzte) als teilerscheinung eimer allgemeinen allergie des neugeborenen, Zischr. Kinderth 1927;43: 630 .

[8] Hodgman JE, Freedman RI, Levan NE. Neonatal dermatology. Pediatr Clin North Am 1971;18(3):71356.

[9] Rudoy RL, Nelson JD. Breast abscess during the neonatal period: a review. Am J Dis Child 1975;129(9):1031-4.

[10] Sonnenschein H, Clark HL, Taschdjian CL. Congenital cutaneous candidiasis in a premature infant. Am J Dis Child 1959;99:97-101.

[11] Pratt AG. Perianal dermatitis of the newborn. AMA Am J Dis Child 1951;82(4):429-32.

[12] Kellum RE, Ray TL, Brown GR. Sclerema neonatorum. Report of a case and analysis of subcutaneous and epidermal-dermal lipids by chromatographic methods. Arch Dermatol 1968;97(4):372-80.

[13] Smith MA, Manfield PA. The natural history of salmon patches in the first year of life. Br J Dermatol 1962;74(1):31-3.

[14] Osburn K, Schosser RH, Everett MA. Congenital pigmented and vascular lesions in newborn infants. J Am Acad Dermatol 1987;16(4):788-92.

[15] Watson W. Selected Genodermatoses. Pediatr Clin N Amer 1978;25(2):263-84.

[16] Wagner AM, Hansen RC. Neonatal skin and skin disorders. Pediatric dermatology. 2nd edn. New York: Churchill Livingstone 1995;112:698-705.

[17] Rivers JK, Frederiksen PC, Dibdin C. A prevalence study of dermatosis in the Australian neonate. J Am Acad Dermatol 1990;23(1):77-81.

[18] Mishra PC, Mathur GP, Mathur S, et al. Normal anatomic variants in the newborn. Ind Pediatr 1985;22(9):649-52.

[19] Dickson J, Yue P. A study of the incidence of neonatal dermatoses. Australas J Dermatol 1979;20:78-81.

[20] LaVoo EJ, Paller AS. Common skin problems during the first year of life. Paediatr Clin $\mathrm{N}$ Amer 1994;41(5):1105-19. 